La conciencia y el problema mente-cerebro

\title{
LA CONCIENCIA Y EL PROBLEMA MENTE-CEREBRO

\author{
Un camino de acercamiento entre la ciencia \\ y la reflexión filosófica
}

\author{
Esteban Fernández Hinojosa \\ Hospital Universitario Virgen del Rocío. Sevilla
}

\begin{abstract}
Resumen: Tanto la ciencia como la filosofía han abordado este problema a lo largo de la historia del pensamiento desde perspectivas epistémicas muy diversas. La primera construyendo hipótesis con objeto de validar modelos teóricos, y la segunda extendiendo su comprensión sistémica al conjunto de lo real, lo que incluye a la actividad científica. La respuesta al problema mente-cuerpo podría ser factible desde una ciencia fundamentada filosóficamente. El postulado ontológico del materialismo emergentista afirma que la mente es una propiedad emergente del cerebro. El postulado epistemológico del realismo científico sostiene que la neurociencia posee legitimidad epistémica para acometer el estudio del proceso mental más genuinamente humano: la consciencia. La complejidad del problema exige un vínculo de conexión entre ambas disciplinas. Se revisan ambos presupuestos (ontológico y epistemológico), y para acotar el presente ensayo dirigimos el arsenal onto-epistemológico al objeto de estudio más apasionante y complejo del cerebro humano: la conciencia. Concluimos que, aunque coherentes, no explican completamente el problema.
\end{abstract}

Palabras clave: Conciencia, autoconciencia, problema mente-cuerpo, método científico.

Consciousness and mind-body problem. A path of rapprochement between science and philosophical meditation

Abstract: Both science and philosophy have addressed this issue throughout history from very different epistemic perspectives. The first, has built hypotheses in order to validate theoretical models, and the second, extends the whole systematic understanding of what is 
real, which includes scientific activity. The answer to the mind-body problem could be feasible in a science grounded in philosophy. The ontological postulate of emergent materialism states that the mind is an emergent property of the brain. The epistemological postulate of scientific realism holds true that neuroscience has epistemic legitimacy to undertake the study of the most genuinely human mental process: consciousness. The complexity of the problem requires connecting links between the two disciplines. In summary, by reviewing both topics (ontological and epistemological), the ontoepistemological arsenal is directed to the most exciting and complex study of the human brain: consciousness. We conclude that a though coherent, it does not explain the main problem of this phenomenon.

Keywords: Consciousness, self-awareness, mind-body problem, scientific method.

Recibido: 24/1/2017 Aprobado: 20/4/2017

\section{Introducción}

La primera parte de la presente revisión es una reflexión construida con el análisis de los fundamentos filosóficos de la neurociencia. Una segunda parte aborda la posibilidad epistémica del estudio científico de la conciencia. Entre los problemas que vertebran la historia del pensamiento occidental, en la confluencia epistemológica entre filosofía y ciencia, uno de los más antiguos, interesantes y poliédricos, es el denominado problema mente-cerebro, almacuerpo en época pre-científica (Armstrong, 1999). Los sistemas filosóficos elaborados por las grandes figuras intelectuales desde la antigua Grecia a la actualidad, que tienen por propósito explicar la realidad in Toto, muestran cómo casi todos ellos formulan una respuesta de carácter ontológico ante los grandes interrogantes que genera el problema, no sólo sobre la existencia real 
de la mente, sino también sobre su dimensión corporal e interacción con el cerebro (Russell, 1968).

Hemos comprobado cómo esta compleja cuestión sigue generando, y en sentido cuantitativo, una ingente producción intelectual. Entre las consecuencias epistémicas destacadas, hay una innovadora corriente de reflexión denominada filosofía de la mente (McLaughlin, 2009). Un área de estudio donde se cruzan diversas disciplinas y se impulsa abundante especulación filosófica de orientación científica, cuyo eje gira en torno al binomio mentecerebro. Una corriente que infiltra los intersticios conceptuales más débiles de la neurología, la psiquiatría y la psicología. Muchas de las teorías elaboradas por la filosofía de la mente centran su reflexión en la naturaleza de la mente, la interacción mente-cerebro y en las ciencias de la salud mental. Asimismo, se reflexiona en torno a las novedosas técnicas de neuroimagen (Fulford, 2013).

Hay dos hipótesis derivadas del presupuesto fundacional que sostiene el encuentro entre ciencia y filosofía:

El materialismo emergentista: propuesta ontológica coherente al servicio de la promoción de programas de investigación teórico-experimentales en neurociencia. Pretendemos mostrar, con brevedad y claridad, el papel que esta propuesta se arroga como condición de posibilidad ontológica de la neurociencia.

El realismo cientifico: resultados experimentales, teorías y modelos conceptuales en neurociencia que pueden confirmar la validez de la propuesta ontológica de dicho materialismo como pretendida solución al problema mente-cerebro. 


\section{Fundamentos onto-epistemológicos}

La ontología y la epistemología configuran históricamente dos de las ramas más relevantes de toda construcción filosófica. La primera trata del estudio de la realidad y de sus características más generales; también de responder a preguntas del tipo: ¿existe el mundo independientemente de nuestro pensamiento? ¿Qué entidades son reales? ¿Es la mente una entidad real? La segunda se dedica al estudio de la cognición, el conocimiento y los constructos conceptuales con los que representamos el mundo. El core de la epistemología es siempre la búsqueda de la respuesta a la cuestión: ¿es posible el conocimiento del mundo?

La denominación filosofía cientifica - que a nosotros nos resulta un tanto confusa - se utiliza para referir una interpretación de la relación cienciafilosofía que exige a esta última, en su calidad de constructora de sistemas racionales que explican la realidad, dos condiciones: compatibilidad con el conocimiento científico validado, y contrastabilidad a través del corpus científico de sus modelos filosóficos (Reinchenbach, 1973). La diferencia entre ambas representaría un valioso elemento de complementariedad, de manera que mientras que la ciencia acota áreas específicas de la realidad y construye hipótesis con el propósito de validar modelos teóricos, la filosofía extiende su arquitectura sistémica al conjunto de lo real, que también incluye la actividad científica. Ambas se enriquecerían en una frontera que halla en la realidad su criterio ontológico de confirmación y encuentra en el conocimiento falible y validable su objetivo cognitivo último (Bunge, 2012). 
De esta guisa, el filósofo contemporáneo quedaría responsabilizado de la maduración de la actividad productiva de la comunidad científica, formulando hipótesis ontológicas de manera tal que sus construcciones conceptuales sean compatibles con los conocimientos validados en neurociencia (Chalmers, 1996). El neurocientífico asumiría la reflexión crítica tanto sobre la condición de posibilidad o existencia real de las entidades, propiedades y procesos que dominan sus modelos y teorías, como sobre la capacidad de su disciplina para describir, explicar y predecir estados presentes y futuros de su objeto de estudio.

Abogamos por una convergencia, entre ciencia y filosofía, que impulse un contexto fecundo para la reflexión interdisciplinar en la ambiciosa y fascinante tarea que trata de descifrar la realidad de la vida.

\subsection{Soluciones planteadas ante el problema mente-cerebro}

Con asombro descubrimos que todas las propuestas filosófico-científicas que han sido enunciadas a lo largo de la historia del pensamiento, sin excepción, se adscriben a uno de los dos grandes modelos (ónticos) de la realidad: el monismo, para el cual la realidad está compuesta de una única sustancia, y el dualismo, que defiende la existencia de dos entidades diferentes e independientes: lo mental y lo material.

\section{Dualismos:}

-Dualismo platónico (animismo): modelo según el cual una entidad espiritual (alma o mente) anima, causa o afecta a la entidad material. Si bien lo material tiende a perecer, el alma — como narra el Sócrates de Platón, en el diálogo Fedón - puede retornar al mundo de las ideas y seguir existiendo. 
Una tesis de inspiración mística. Se ha dicho que "toda la obra de Henri Bergson, precursor del antipositivismo, está recorrida de un dualismo radical" (Gomá, 2003).

-Paralelismo psicofísico: la mente y la materia son dos entidades autónomas e independientes, cuya actuación se da en paralelo y de modo sincronizado, pero sin interacción real entre ellas. Entre los siglos XVII y XVIII, el matemático y filósofo Gottfried Leibniz sostuvo esta concepción, postulando que el alma y el cuerpo no sólo existen de forma independiente, sino que están gobernadas por leyes distintas. A la luz del actual conocimiento científico, no resulta posible formular hipótesis neurocientíficas que puedan ser contrastadas bajo este presupuesto.

-Epifenomenalismo: todo proceso mental es un epifenómeno o fenómeno dependiente de un proceso cerebral (Caston, 1997). Entre los primeros defensores de este modelo se encuentra el biólogo evolucionista T. H. Huxley. La forma en la que se relaciona el fenómeno dependiente (la mente) con el hecho del que depende (el cerebro) no está aclarado.

-Dualismo interaccionista: mente y cerebro son dos sustancias diferentes y autónomas que pueden interactuar entre sí. Aunque la primera formulación sistemática de esta propuesta se debió a René Descartes, su actualización fue establecida en los años 70 y 80 del siglo XX (Eccles, Popper, 1987). Las hipótesis que tratan de explicar tal interacción no alcanzan estatus epistémico lo suficientemente coherente como para ser adaptadas a ningún modelo neurocientífico (Damasio, 1994).

Monismos: 
-Idealismo: toda la realidad es mental o ideal. Su tesis no encuentra acomodo en el método científico. La obra filosófica que más extensamente recoge el dictum idealista es La fenomenología del espiritu de Hegel.

-Monismo neutral: la realidad está constituida por una sustancia neutral. La ambigüedad de este modelo imposibilita traducirlo a enunciados teóricoexperimentales.

-Materialismo eliminativo o conductismo filosófico: los enunciados sobre lo mental carecen de sentido, bien porque la mente no tiene existencia real —nada es mental - (conductismo ontológico), o porque, al no ser posible emitir un juicio sobre su existencia (conductismo metodológico), la ciencia evita hacer uso de enunciados que la asuman como objeto de estudio. Watson y Skinner promovieron el desarrollo de la psicología científica sobre este pilar ontológico; sin embargo, el modelo quedó sesgado por su incapacidad para conceptualizar los procesos cognitivos (Dennett, 1987).

-Materialismo fisicalista o teoría de la identidad: la mente es el cerebro. Los conceptos mente y cerebro hacen referencia a una misma y única entidad que presenta - al igual que toda entidad real - una estructura exclusivamente física. Al reducir la realidad in toto a la dimensión física, el modelo no explica los procesos evolutivos en las distintas especies biológicas. Los trabajos que mejor ilustran la teoría de la identidad pertenecen a los filósofos australianos U.T. Place y J. C. Smart, y al alemán H. Feigl.

-Funcionalismo: los estados mentales son estados funcionales; su acontecer es independiente de los estados cerebrales concretos de los que derivan. Tal función (software) puede ser implementada por otro soporte físico no cerebral (hardware). Concede primacía a la función cognitiva, y abandona de 
modo no científico el estudio del sistema nervioso. Sus principales representantes son H. Putnam, J. Fodor y D. Lewis. La asunción ad litteram de esta tesis obliga al científico a sustituir el estudio del cerebro humano por el estudio de sistemas computacionales abstractos.

-Emergentismo: la mente ha emergido de la evolución cerebral. El referente ontológico actual del proyecto científico-filosófico es el materialismo emergentista (Bunge, 1977), que sintetiza las dos dimensiones de la filosofía científica.

\subsection{Materialismo emergentista}

Respecto del problema mente-cerebro, M. Bunge defiende los siguientes postulados de su ontología científica.

Primer postulado: "Un objeto es real (tiene existencia real independientemente de la actividad cognitiva del observador) si, y sólo si, es un objeto material. Ergo la realidad es el conjunto de todos los objetos materiales”. De tal postulado se deduce que la mente, para tener existencia real, o es un objeto material, o pertenece, como componente o propiedad, a un determinado objeto material.

Segundo postulado: "Todo objeto real es un sistema o un componente de un sistema”. Si el primer postulado niega la posibilidad de que la mente fuera un objeto real, en este segundo se deduce que no es un sistema. El corolario de este postulado es que si la mente no es ni un sistema (redes neuronales) ni un componente de un sistema (neurona) sólo resta la posibilidad de que se trate de la propiedad de un sistema. 
Tercer postulado: "Todo sistema posee al menos una propiedad emergente”. Respecto del problema mente-cerebro, defiende que lo mental no es una propiedad de origen inmaterial, sino una propiedad emergente o novedad cualitativa de sistemas neurobiológicos altamente complejos y producto de la evolución. Se constituye existencialmente como propiedad de un objeto real o material, esto es, como propiedad material y, sin embargo, no como propiedad física en sí. Así, las propiedades neurobiológicas del cerebro están estructuradas con la singularidad de no poder ser reducidas a las leyes de la física. Sería un sofisma epistémico explicar la estructura de un nivel de la realidad (el neurobiológico) con leyes de otro (el cuántico) (Bunge, 2002). Así, los estados mentales son propiedades emergentes de cerebros altamente evolucionados (proceso onto-filogenético) y no pueden ser identificados con el cerebro. Contra la teoría de la identidad, Bunge defiende que no son equiparables mente y cerebro, como no lo son estómago y digestión, o pulmones y respiración. El corolario de este último postulado es que todos los procesos mentales lo son en los cerebros superiores. Y son emergentes con respecto a los componentes celulares del cerebro. El materialismo emergentista trata de armonizar la defensa de una única sustancia autónoma (materia o cerebro) con la demostración de ciertas propiedades emergentes de un sistema. Propiedades que - como ha modelizado la neurobiología evolutiva - son novedades cualitativas evolutivamente desarrolladas de sistemas biológicos concretos (como las funciones ejecutivas). 


\subsection{Realismo científico}

Una vez planteada la ontología científica del materialismo emergentista, valga la siguiente incursión en el campo de la filosofía de la naturaleza para interpretar las teorías, modelos y conceptos de la neurociencia actual, sin entrar in extenso en el actual debate (Miller, 2006) entre las dos corrientes de mayor predicamento sobre la relación entre conocimiento científico y mundo exterior: el realismo científico y el instrumentalismo científico.

El instrumentalismo científico sostiene que el objetivo fundamental de la teorización científica es "salvar los fenómenos" mediante los constructos teóricos que mayor simplicidad exhiben (Van Fraassen, 1980). Por tanto, el objetivo de la empresa científica radica en el éxito empírico a la hora de predecir, descubrir o intervenir en la naturaleza. Las teorías, los modelos y los conceptos de la ciencia no describen ni representan la realidad. En el nivel teórico, la verdad es tan inalcanzable como innecesaria. Si no hay representación de la realidad ni viabilidad para la aproximación a la verdad, tampoco habrá posibilidad de progreso científico más allá del pragmatismo. El más destacado representante de esta escuela es el filósofo de origen holandés Bastiaan Van Fraassen, que sostiene un tipo de anti-realismo denominado empirismo constructivo.

En cuanto a las tesis del realismo científico, estas son adoptadas como concepciones válidas del conocimiento científico, pues sin su concurso no sería posible cuadrar (epistémicamente) el materialismo científico de cara a la construcción teórico-experimental de la neurociencia. Argumentan sus defensores que con los principios teóricos del instrumentalismo científico el neurocientífico tendría que aplazar sine die su respuesta a la pregunta: ¿existe 
el cerebro? Dado que sus teorías, modelos y conceptos tienen como finalidad epistémica "salvar los fenómenos", entonces tanto cerebro como mente serían categorías ontológicas vacías, lo que las deslegitima para emitir un juicio científico sobre su existencia. De tal argumentación se infiere que la entidad cerebro se diferencia de la entidad mente sólo por su utilidad y no por su ajuste a la realidad.

La propuesta general de los realistas científicos (M. Devitt, M. Bunge, K. Popper, y un largo etc.) se resume en las siguientes tesis:

1. La realidad es ontológicamente independiente de la actividad cognitiva del sujeto cognoscente. Tanto el cerebro de un sujeto concreto como los procesos neurocognitivos emergentes de tal cerebro, son independientes de la actividad cognitiva de otro sujeto; su existencia (como realidad) no depende de que un segundo sujeto piense en ellos.

2. La verdad es una relación semántica entre el lenguaje y la realidad. El realismo científico integra una versión actualizada de esta teoría de la correspondencia (Tarski, 1944), con la convicción de que la metodología científica es la mejor guía en la búsqueda sistemática de la verdad. La neurociencia ha afinado su lenguaje a medida que, mediante el método científico, iba obteniendo información empírica sobre su objeto de estudio. Su condición teórico-experimental posibilita la relación lenguaje-realidad.

3. Los conceptos de verdad y falsedad son aplicables a los productos de la actividad científica, como leyes, modelos o teorías. Las proposiciones sobre la existencia de las entidades teóricas como cerebro, mente, corteza prefrontal... tienen valor de verdad (pueden ser verdaderas o falsas). De esta 
guisa, la neurociencia actual declara como falsa la afirmación: la mente existe independientemente del cerebro.

4. La verdad es un objetivo esencial de la actividad científica. La neurociencia aspira a que sus modelos, teorías y conceptos sean aproximadamente verdaderos; digan algo verdadero acerca de cómo es la realidad.

5. La verdad no es fácilmente accesible o reconocible (Bunge, 2001). Incluso nuestras mejores teorías pueden ser falsas. Un repaso a la historia del estudio del sistema nervioso central aporta sobrados ejemplos que ilustran este hecho.

6. ¿Cómo podemos explicar el éxito práctico de la ciencia (único elemento relevante para el pragmatismo y el instrumentalismo científico)? Asumiendo que las teorías científicas son aproximadamente verdaderas o suficientemente cercanas a la verdad. El hecho de que las teorías y modelos neurocientíficos de hoy se aproximen más a la verdad que en otras épocas permite enunciar proposiciones racionales del progreso cognoscitivo o defender que el uso sistemático de métodos experimentales auto-correctores posibilita el progreso neurocientífico (Gazzaniga, 1995).

\section{Sobre la conciencia}

En lo que resta de la presente exploración sobre el acercamiento cienciafilosofía, abordaremos la aptitud del materialismo emergentista para ser postulado como condición de posibilidad ontológica de la actual neurociencia, y si sus resultados, sus teorías y sus modelos son, en las tesis del realismo científico, válidas como solución al problema mente-cerebro. Enfocaremos el 
arsenal onto-epistemológico sobre la conciencia, el fenómeno más complejo y apasionante del estudio actual sobre el cerebro humano.

¿Es la conciencia un fenómeno biológico resultado del funcionamiento cerebral, como lo es el lenguaje, la memoria o el arte? Una diferencia fundamental con esas categorías sería la siguiente: la conciencia se halla inextricainextricablemente vinculada a la subjetividad. Ella es acto privativo de cada sujeto, cada uno percibe singularmente cómo es y lo que siente. Si se nos pregunta cómo somos o qué sentimos, podemos responder con una narración sobre nuestras experiencias internas en primera persona, algo que resulta imposible si se nos pregunta qué siente nuestro amigo.

Se trata de una propiedad de alto nivel que se manifiesta a través del sistema nervioso. En el marco de relación entre la conciencia y el sistema nervioso, se defiende que los procesos neuronales de nivel inferior son causa de la experiencia consciente, mientras que la conciencia es una propiedad de alto nivel de un sistema que se compone, asimismo, de elementos neuronales de nivel inferior. Los procesos cerebrales de alta complejidad tendrían propiedades semejantes a las de las moléculas que forman los seres vivos, y la mente humana propiedades emergentes respecto de las neuronas que conforman el cerebro. Es la tesis de Searle y Gazzaniga. Sin embargo, Bunge insiste en hablar de fenómenos mentales, y es ahí donde el materialismo emergentista nos resulta más discutible: si se postula que los hechos mentales no son afecciones de una sustancia inmaterial, sino estados o procesos que se presentan en organismos naturales, entonces es compatible con las ciencias naturales y, en consecuencia, es posible aplicar el método científico a la investigación de los hechos mentales. He aquí un encuentro entre filosofía de 
la naturaleza y neurociencia. Declaramos nuestra duda abierta sobre el hecho de que el análisis neurocientífico (que incluye la dimensión psíquica) sea condición suficiente para explicar la intrincada complejidad del ser humano, pero a pesar de esta insuficiente condición, abogamos por dicho análisis como acercamiento a la complejidad de la mente, sobre la base de sus componentes psicológico y neurológico. En el afán por comprender un fenómeno de tal envergadura, se hace necesario establecer ciertas líneas maestras, como sus propiedades, niveles de complejidad, o cómo sus componentes se organizan para formar un sistema de mayor complejidad. Como demuestra el trabajo de laboratorio, este fenómeno no se explica en su integridad con el solo conocimiento del funcionamiento específico de moléculas, neuronas, sinapsis y neurotransmisores. Los procesos mentales pueden ser abordados desde distintos niveles de análisis, como los micro-moleculares (neurotransmisión o neuronas), macro-moleculares (filosofía o psicología evolucionista), u otras escalas de observación. No obstante, en base al principio de plausibilidad biológica, admitimos que los niveles macro puedan soportar e integrar los conocimientos que llegan desde los niveles inferiores. $\mathrm{Y}$ aunque apostamos por que los niveles de análisis que persiguen tantear realidades tan complejas no sean excluyentes sino integradores, sin embargo y contra lo sostenido por Wagensberg — posiblemente para otras épocas-, creemos que en el actual estadio de la cultura es un anacronismo rellenar las grietas del conocimiento con la pasta de la ideología.

Conceptos como conocimiento, atención, percepción, vigilia, memoria, motivación, sensación, emoción o funciones ejecutivas, se entretejen para gestar la urdimbre de la conciencia. Pero no dejan de ser figuras demasiado 
vaporosas y diversas para constituir su única realidad. En sentido lato, el castellano presenta una división conceptual de elevado interés para abordar este capítulo, al diferenciar entre los verbos ser y estar. Estar consciente habla de estados en los que se está despierto. Se tiene conciencia cuando se está despierto y se pierde en el sueño profundo o bajo anestesia, para recuperarse de nuevo al despertar. Ser consciente consiste en la capacidad de percepción de uno mismo en términos relativamente objetivos, aun manteniendo un sentido de subjetividad. Se trata de una paradoja natural de la mente humana, que intenta alcanzar objetividad, es decir, percibir un objeto o una situación o incluso una interacción, de una forma que se parezca a la percepción de otros, manteniendo al mismo tiempo un sentido de interpretación privada, subjetiva y única de la experiencia. Dicho carácter paradójico de la conciencia desvela un estado emocional simultáneo al proceso cognitivo. La conciencia humana implica, en consecuencia, una integración de las dimensiones cognitiva y emocional.

Estar y el ser consciente, coincide con lo que el filósofo de la mente David Chalmers denominó como "el problema blando" y "el problema duro" de la conciencia. El primero incluye aspectos como la vigilia, la atención o el conocimiento, y el segundo hace referencia a otros tan complejos como la autoconciencia o la cognición social. Así las cosas, el concepto de conciencia como idea unitaria plantea dificultades de acercamiento a su realidad. El problema blando se resuelve al verse satisfecho con modelos experimentales del funcionamiento cerebral, que permiten establecer vínculos entre actividad cerebral y comportamientos. En cambio, el problema duro exige explicaciones en las cuales la relación cerebro-mente no puede ser computada de forma 
algorítmica y, por tanto, se presta a mayor nivel de especulación. Para Chalmers, el estudio de la conciencia desde la perspectiva de problema fácil se inscribe en las investigaciones que cumplen con el reduccionismo materialista y funcionalista desde la óptica de tercera persona, y por tanto, puede ser integrado en el método científico convencional. Entre los problemas fáciles como materia de investigación científica, este autor destaca: la capacidad de discriminar, categorizar y reaccionar a los estímulos del medio ambiente, la integración de la información por un sistema cognitivo, la información de los estados mentales, la capacidad de un sistema para acceder a sus propios estados internos, el foco de la atención, el control deliberado de la conducta, y la diferencia entre el sueño y la vigilia.

Esta distinción también coincide con la establecida por el Premio Nobel Gerald Edelman entre conciencia primaria y conciencia de orden superior o secundaria. La primaria se encuentra también en otras especies animales con estructuras neurológicas similares a la humana, y se da en el sueño REM. Se define como la capacidad para construir escenas mentales vívidas del presente, con acceso muy restringido al pasado y al futuro. Es la conciencia Truman Show del espectador pasivo, que ve en vivo y en directo la trama de su realidad. Una conciencia sin piloto. En la conciencia secundaria o de orden superior, propia del ser humano, se hospeda un sentido de la identidad propia y una capacidad explícita para construir escenas pasadas y futuras, además de requerir de una elevada capacidad lingüística. 
La conciencia y el problema mente-cerebro

\subsection{La experiencia consciente}

En el sistema nervioso activado se habilita la capacidad de percibir diversidad de estímulos, tanto del medio interno como del ambiente, y generar la experiencia consciente. ¿Qué hay entre el estímulo y la experiencia consciente? Diversos autores defienden que la experiencia consciente es una propiedad emergente del sistema nervioso. Hoy se cuenta con modelos que facilitan la comprensión de tales estados intermedios radicados entre el estímulo y esa experiencia consciente. Este nivel continúa en la parcela de los problemas fáciles de la conciencia, pero es requerido para abordar los niveles de mayor complejidad.

Se habla de experiencia consciente, como de una forma especial de proceso surgido de la estructura y funcionamiento dinámico del cerebro, lo que ha derivado en una serie de propiedades fundamentales. Edelman y Tononi sostienen que dos de estas propiedades son que la experiencia consciente es integrada, esto es, no puede subdividirse en componentes independientes, $\mathrm{y}$ que se encuentra altamente diferenciada, o sea, son experimentados diversos estados conscientes. Hay aspectos fundamentales de la experiencia consciente comunes a todas sus manifestaciones fenomenológicas: es individualizada, se encuentra unificada, es coherente y puede ser diferenciada.

F. Crick defiende en La búsqueda científica del alma que la clave radica en conocer el comportamiento de las neuronas asociadas con la experiencia consciente. En neurociencia es necesaria una determinada actividad neuronal asociada a una experiencia consciente. En cada momento, la conciencia se corresponde con un tipo concreto de actividad de un grupo pasajero de neuronas, que forman parte del amplísimo conjunto de candidatas 
potencialmente partícipes. En ese libro, Crick se centra en la percepción visual y señala que cualquier objeto concreto del campo visual es representado por el disparo de un grupo de neuronas. Cada objeto presenta características diferenciadas - forma, color, movimiento, textura- que son procesadas en áreas visuales especializadas en cada característica.

Los trabajos de Logothetis en percepción visual consciente, han recreado situaciones experimentales en las que una foto unas veces es reconocida y otras no, siendo la foto - el estímulo que impregna la retina- exactamente la misma. Cabe preguntarse si las neuronas responden siempre de igual manera ante el mismo estímulo, o si responden a la percepción consciente activándose sólo cuando la foto es reconocida. Los experimentos en monos de Kanwisher han demostrado que las neuronas de la corteza visual primaria responden al estímulo, con independencia de si es reconocido o no, mientras que las neuronas de las áreas que desarrollan un procesamiento visual más avanzado, como las de la corteza ínfero-temporal, tienden a modular su actividad dependiendo del reconocimiento del objeto. En humanos, los experimentos de este mismo autor sobre percepción consciente se han limitado a estudios con resonancia magnética funcional. La limitación de estos estudios radica en que la información del comportamiento neuronal que ofrece dicha técnica es, por el momento, vaga, difusa e indirecta. De esta guisa, el conocimiento actual de los correlatos neuronales de la percepción consciente se hallan limitados tanto por la imposibilidad de observar directamente la actividad neuronal, como por el desconocimiento sobre cómo los resultados en monos pueden ser extrapolados a humanos. Existen casos de pacientes epilépticos en los que se han implantado electrodos intracraneales 
con objeto de delimitar el foco epileptógeno y valorar posibilidades de abordaje quirúrgico. Este procedimiento permite monitorizar directamente la actividad neuronal en el cerebro humano. El grupo de Quian-Quiroga ha mostrado neuronas del hipocampo respondiendo de manera abstracta ante la persona que es presentada: unas neuronas respondieron sólo a distintas fotos de la actriz Jeniffer Aniston, otras a fotos solamente de Halle Berry, e incluso a su nombre escrito.

El problema acerca de cómo las neuronas, correspondientes a diferentes áreas visuales, se activan temporalmente como una unidad se conoce como problema del enlace. En el cerebro del observador, la imagen fragmentada de un objeto se analiza en función de sus diversos atributos, color, forma, contraste, etc. Cuando el sujeto percibe un objeto, se activan columnas de células localizadas en distintas regiones de la corteza visual en respuesta a cada atributo de la imagen. La estrategia de reconstrucción de la imagen fragmentada se basa en la activación simultánea de las neuronas implicadas en el análisis de los atributos mostrados por cada objeto, de manera que la percepción de dicho objeto sea coherente en cada instante. Así que "ver" un objeto implica la activación de varios grupos de neuronas en diferentes áreas del sistema visual y de manera correlacionada. El problema del enlace consiste en comprender cómo estas neuronas, que detectan distintos atributos del objeto, se activan temporalmente de manera sincrónica, y cómo se disparan de manera correlacionada, sobre todo si se percibe simultáneamente más de un objeto.

La hipótesis del enlace postula que éste resulta del disparo correlacionado, cíclico y oscilatorio de todas las neuronas implicadas en la percepción de un objeto. Se han registrado oscilaciones periódicas en la actividad eléctrica de la 
corteza cerebral visual de gatos a los que se les mostraba un objeto. Estas oscilaciones mostraban una frecuencia media de $40 \mathrm{~Hz}$, lo que representa la actividad promedio del disparo correlacionado de las neuronas visuales de la vecindad de los sitios de registro. Se considera que las oscilaciones de $40 \mathrm{~Hz}$ en la corteza visual son la base neurológica del enlace. Distintos grupos proponen que el disparo de las neuronas especializadas en la detección de cada atributo de un objeto percibido se enlaza en el tiempo por medio de un mecanismo que las sincroniza unas con otras. A partir de esta idea, Crick y Koch han postulado que las oscilaciones de $40 \mathrm{~Hz}$ pueden ser el correlato neuronal de la conciencia visual. La hipótesis es coherente, aunque no ha sido demostrada.

La unidad de percepción debe de tener lugar en múltiples módulos de percepción diferenciados, aunque hay un biato entre el conocimiento perceptivo y su significado, de ahí que se plantee la nueva cuestión acerca de cómo se dota de significado esta unidad de percepción. Se deduce que dicha cualidad guarda relación con el campo de proyecciones de la neurona o patrón de conexiones sinápticas con otras neuronas que codifican explícitamente los conceptos asociados. De esta manera, las neuronas que perciben un objeto están conectadas con otras que expresan el nombre del objeto, sus características generales, los recuerdos vinculados a ese objeto y sus tonos emocionales.

Nos sumergimos aquí en el profundo y anchuroso océano del problema duro de la conciencia, el estudio de las características que otorgan a una red neuronal capacidad de generar significado. La hipótesis del enlace no explica el problema del enlace en las percepciones sensoriales polimodales. Y lo que resulta trascendente, tampoco resuelve el cómo y quién sincroniza el disparo 
de las neuronas implicadas en la percepción de un objeto, toda vez que estas se encuentran localizadas en áreas distintas y distantes en el sistema nervioso. En un intento de superar estas limitaciones, Roberto R Llinás postula que el cerebro es un sistema cerrado capaz de generar sus propios ritmos, basado en las propiedades eléctricas intrínsecas de las neuronas que lo componen y sus conexiones. Basándose en estudios de magneto-encefalografía, este autor sostiene que el sistema tálamo-cortical (que comprende regiones talámicas específicas, como el complejo ventro-basal, e inespecíficas, como los núcleos intralaminares o de la línea media junto a sus conexiones con la corteza), constituyen el sustrato físico del conocimiento. Para Llinás las oscilaciones de $40 \mathrm{~Hz}$ registradas en áreas corticales resuenan con las oscilaciones neurales en los núcleos talámicos. Estas oscilaciones progresan desde el polo frontal al occipital con un barrido de 12-13 ms de duración, período en el que el cerebro procesa un suceso simple o quantum, por lo que la experiencia consciente podría ser una sucesión de quanta de tiempo. Si los circuitos activos cerebrales barren la corteza cada $12 \mathrm{~ms}$, nuestro conocimiento se produce por el acoplamiento temporal de los quanta de conocimiento que proporciona cada barrido cerebral.

Otra hipótesis interesante sobre la experiencia consciente y que comparte con las de Crick y Llinás la convicción de que es imprescindible conocer el funcionamiento cerebral para comprender la conciencia, es la llamada hipótesis del núcleo dinámico de Edelman y Tononi. Para ellos la aproximación al problema de la conciencia se resolvería de forma más adecuada enfocándose sobre sus propiedades fundamentales, ya comentadas, de la integración, por la que una experiencia consciente no puede ser dividida, y la diferenciación, 
pues se vivencian millones de experiencias conscientes, y explicándolas en términos de procesos neuronales. Hacer referencia a las propiedades de la experiencia consciente exige explicar estos procesos neuronales distribuidos, antes que las propiedades específicas de cada neurona. En este sentido, la hipótesis contiene dos aspectos destacables; por un lado, se sostiene que un grupo de neuronas puede contribuir a la experiencia consciente sólo si forma parte de una agrupación funcional distribuida que, a través de interacciones de reentrada en el sistema tálamo-cortical, alcanza un alto grado de integración en unos centenares de milisegundos. $Y$ por otro lado, se señala que para sustentar la experiencia consciente es necesario que este grupo funcional se encuentre altamente diferenciado, lo que significa alto grado de complejidad. Es necesario aclarar dos conceptos básicos en la hipótesis de Edelman y Tononi: el de reentrada y el de complejidad de sistema. Este último se refiere a la cantidad de estados diferentes que puede experimentar un grupo funcional o proceso neuronal unificado, lo que requiere diversidad de elementos especializados y conexiones no azarosas entre ellos. El problema de enlace planteado por Crick es solucionado en esta hipótesis con el concepto de reentrada, que alude al proceso por el cual un elevado número de neuronas interaccionan rápida y recíprocamente. Por reentrada se entiende el intercambio recursivo y continuo de señales paralelas entre áreas del cerebro recíprocamente conectadas. Entre sus consecuencias destaca la sincronización general de la actividad de distintos grupos de neuronas distribuidas entre numerosas áreas funcionalmente especializadas del cerebro. El disparo sincronizado de neuronas dispersas que se encuentran conectadas por la reentrada es la base de la integración de los procesos de percepción y motores. Esto demostraría que la 
experiencia consciente no tiene lugar en una concreta localización del cerebro. Se trataría de un proceso coherente, resultado de interacciones entre grupos neuronales distribuidos por diferentes áreas, y que produce la integración rápida (100-250 ms) después de la presentación del estímulo.

Esta hipótesis (según la cual existe un gran conjunto de sub-grupos neuronales que en cientos de milisegundos se integran y forman un proceso neuronal unificado de alta complejidad), decíamos que es conocida como hipótesis del núcleo dinámico. Núcleo, por su alto grado de integración, y dinámico, por su estado de cambio continuo. Así las cosas, lo que subyace a la experiencia consciente es un proceso neuronal ampliamente distribuido y con intervención del sistema tálamo-cortical, que además de resultar un proceso integrado exhibe una elevada diferenciación, dado que sus patrones de actividad se hallan en constante cambio.

Damasio denomina proto-self a la colección coherente de pautas neuronales que representan, momento a momento, el estado de la estructura corporal en sus múltiples dimensiones. Tanto los receptores de la musculatura esquelética como los del tacto, contribuyen a otra función del sistema nervioso, la de informar al organismo sobre lo que acontece en el mundo exterior para que actúe en consonancia. El sistema tálamo-cortical, utilizando la información que proporcionan los sentidos, organiza y categoriza percepciones y programa la actividad motora. Así que la percepción de cualquier objeto implica una alteración del estado corporal que activa los sistemas de valoración del cerebro y la memoria, y hace posible el aprendizaje y la categorización conceptual. 
En resumen, los referidos autores Crick, Llinàs, Damasio, Edelman y Tononi coinciden en que la experiencia consciente es el resultado de la integración de la actividad de poblaciones de neuronas que producen el acoplamiento de las respuestas, en áreas corticales distribuidas, hasta alcanzar la sincronización que les dota de coherencia global.

\subsection{Autoconciencia}

La función neuronal constituye un nivel ineludible de análisis en el estudio de la experiencia consciente, sin embargo parece fútil en el estudio del problema duro de la conciencia: la autoconciencia.

Stuss y Benson definen la autoconciencia como un atributo humano que no sólo permite la conciencia de uno mismo, sino que también percibe la posición de uno en su entorno. En su cometido controla la propia actividad mental, representa las experiencias actuales en relación con las del pasado, hace útil el conocimiento adquirido y guía la toma de decisiones para el futuro. George Prigatano la define como "la capacidad de percibirse a uno mismo en términos relativamente objetivos manteniendo un sentido de subjetividad" (Prigatano, 1991). Así por ejemplo, de cualquier lectura necesitamos saber, por una parte, que nuestra comprensión del texto se acerca a la de otros lectores, pero también es preciso poseer una interpretación personal del mismo. Se trata de la comentada paradoja de la autoconciencia: necesitamos percibir el mundo de una forma similar a la percibida por otros, y al mismo tiempo un sentido de interpretación privada, subjetiva y singular de esa experiencia. Este aspecto de la autoconciencia implica un proceso cognitivo a la vez que un estado emocional. 
Antonio Damasio ha propuesto la división de la conciencia en conciencia central y conciencia ampliada o extendida. La primera proporciona al organismo la sensación de ser en un tiempo (ahora) y en un lugar (aquí). El alcance de la conciencia central es el aquí y el ahora, por lo que presenta similitudes con la experiencia consciente analizada en los párrafos anteriores. No obstante, esta conciencia central no es coincidente con la autoconciencia, ya que ni accede al pasado, ni se proyecta al futuro. Pero el tipo complejo de conciencia, a la que denomina conciencia ampliada o extendida, proporciona al organismo la sensación elaborada de ser, esto es, una identidad, y sitúa al sujeto en un punto de su devenir autobiográfico, consciente del pasado vivido y del futuro anticipable, a la vez que lo hace conocedor del mundo que le rodea.

La conciencia ampliada es la consecuencia de la capacidad de registrar experiencias y la de reactivar dichos registros de manera que, como objetos, generen la sensación de "ser yo el que conoce". De ahí que pueda considerarse la capacidad de ser consciente de un extenso ámbito de entidades y sucesos, lo que ofrece un sentido de perspectiva. Con estas premisas, se puede decir que generamos pulsos de experiencia consciente para un objeto $y$, a su vez, un conjunto acompañante de recuerdos autobiográficos reactivos, sin los cuales no tendríamos sensación de pasado ni de futuro ni, en consecuencia, existiría continuidad histórica (autoconciencia).

En cuanto a la base anatómica de la consciencia ampliada, Damasio sugiere un espacio de imágenes en el que se yuxtaponen los contenidos mentales de la experiencia consciente con un espacio de disponibilidad en el que los recuerdos contienen registros de conocimiento implícito. Los ele- 
mentos fundamentales de la autoconciencia son los que se corresponden con nuestra identidad, con nuestras experiencias pasadas y expectativas. Para este célebre neurólogo, la autoconciencia es un proceso de activación coordinada y exhibición de recuerdos personales, situados en una red ubicua. Los elementos esenciales surgen de una red ubicua que se activa incesantemente y se localiza en zonas de convergencia de las cortezas superiores temporales y frontales y en núcleos subcorticales, como la amígdala, y cuya activación es coordinada por diversos núcleos talámicos, mientras que el mantenimiento de los componentes reiterados durante períodos más amplios exige el concurso de la corteza prefrontal.

$\mathrm{Al}$ referirnos al ser consciente, resulta de interés la referencia a dos tipos de autoconciencia que comparten su contenido experiencial y fenomenológico: la consciencia noética y la autonoética (Tulving, 1981). La primera es evolutivamente más antigua y, de algún modo, se relaciona con el sistema de memoria semántico. Es resultado de la interacción entre la experiencia presente y la memoria del pasado relacionada con el saber (no con el recordar). En cambio, la consciencia autonoética es filogenéticamente más joven y se apoya en la memoria episódica, por lo que puede recordar el pasado, a la vez que proyectar hacia el futuro. Cuando recordamos el pasado, somos autonoéticamente conscientes de ese pasado. Esta consciencia autonoética incluye y, a su vez, trasciende a la consciencia noética. Ambas están determinadas por las propiedades del cerebro individual y por el estado fisiológico de cada momento, de ahí que puedan presentar una enorme variabilidad interindividual. Autoconciencia significa poseer una experiencia particular que depende 
tanto del estado general del estar consciente como de los estímulos procedentes del mundo interior y exterior, que son únicos.

Tulving ha añadido en 2002 el concepto decronestesia para referirse a la capacidad cognitiva de expresar la conciencia en una dimensión temporal relacionada con su propia existencia y la de los demás (tiempo subjetivo). Hace referencia al recuerdo de los hechos de la vida, además de la planificación del futuro. Coincide con la consciencia autonoética, en que también implica la conciencia del yo en el tiempo, pero el énfasis en los conceptos de yo y tiempo difieren. En la autonoesis, lo importante es la conciencia del yo, mientras que en la cronestesia lo es el tiempo subjetivo. Esta distinción, aparentemente banal, arroja luz en la práctica clínica de pacientes con recuerdos que no pueden situar en el tiempo o que se muestran incapaces de situar sus recuerdos y planes en un tiempo subjetivo.

El sustrato anatómico de la conciencia autonoética y de la cronestesia se relaciona con la corteza prefrontal. La evidencia clínica sugiere que las lesiones en el cerebro derecho anterior afectan a ambas conciencias con mayor preservación de la memoria semántica (conciencia noética). Algunos estudios con neuroimagen funcional han comparado las redes neurales que se activan cuando a un sujeto se le proporciona información autobiográfica o información ficticia, mostrando que los recuerdos autobiográficos activan la amígdala derecha y la corteza pre-frontal ventral derecha.

La mayoría de trabajos revisados se refieren a las propiedades emergente del cerebro, toda vez que elevados niveles de consciencia sólo se dan en cerebros filogenéticamente más evolucionados. Señalan a la corteza prefrontal como sede de la autoconciencia. Anomalías en la ínsula y regiones mediales 
de la corteza prefrontal, como el cíngulo anterior, orbito-frontal y regiones subcorticales, se han relacionado con ella. Son varias las razones que apoyan esta localización:

- Recibe señales procedentes de todas las regiones sensoriales en las que se forman las experiencias conscientes, incluidas las imágenes que forman parte de nuestros pensamientos.

-También de las cortezas somato-sensoriales que representan los estados corporales pasados y actuales.

-Recibe señales de áreas bio-reguladores como los núcleos troncoencefálicos y del prosencéfalo basal, de la amígdala, el cingulado anterior y el hipotálamo.

- Representa categorizaciones de las situaciones en las que el organismo ha estado involucrado, es decir, clasifica contingencias de la experiencia vital.

El grupo de Allman ha relacionado la ínsula con la conciencia interoceptiva y emocional, la empatía y la conducta social cooperativa. En el cíngulo anterior y en la corteza fronto-insular, se encuentran las neuronas de von Economo, que sólo aparecen en especies que se identifican ante un espejo y poseen estructura social, como chimpancés, delfines o elefantes. La muerte de estas neuronas se relaciona con pérdida de conciencia emocional y con alteraciones de conducta en demencias fronto-temporales.

¿Qué diferencias y qué coincidencias existen entre las narrativas de pacientes sin autoconciencia? El neurocientífico V. Ramachandran plantea que una creencia no es necesariamente unitaria: es posible que el autoengaño se encuentre en el hemisferio izquierdo, mientras que el derecho continúa sabiendo la verdad. Para este autor, la clave del autoengaño radica en la división 
del trabajo entre los dos hemisferios cerebrales y en la necesidad de crear sensación de coherencia y continuidad en nuestras vidas. Cada uno de los hemisferios se encuentra especializado en diferentes capacidades mentales; la asimetría funcional más notable corresponde al lenguaje. Además de estas divisiones funcionales, Ramachandran sugiere que existe una diferencia aún más fundamental, el estilo cognitivo de cada hemisferio, que puede contribuir a explicar las modalidades de negación y de falta de conciencia.

El cerebro humano puede quedar abrumado, en cualquier momento, por una cascada de información continua que debe ser integrada coherentemente en la perspectiva de la propia imagen y de la que esperan los demás. Para poder generar acciones coherentes o mantener un determinado status quo, el cerebro debe disponer de algún mecanismo que permita cribar esa información y ordenarla en un esquema de creencias estable y con consistencia interna. De integrar la información en la imagen previa del yo.

Las estrategias de adaptación empleadas por cada hemisferio son diferentes. La del izquierdo consiste en crear un sistema de creencias y encajar toda nueva experiencia en él. Con la información incoherente recurre a la negación, reprimiendo e inventando una historia que permita mantener su statu quo. El hemisferio derecho, en cambio, actúa poniendo en tela de juicio ese statu quo buscando inconsistencias globales. Cuando la información anómala alcanza cierto umbral, este hemisferio lleva a cabo una revisión global del modelo e impone su cambio. Este umbral es específico en cada sujeto y depende de aspectos tan diversos como los rasgos de personalidad o el tipo de experiencia. 
Estos modelos de autoconciencia (Stuss, Damasio, Tulving) difieren en el modo de acercarse a la compleja realidad del yo, pero aportan dos hechos fundamentales para establecer un discurso con base en la neurociencia. Por un lado, sitúan la autoconciencia en el cerebro en un intento de acabar con la dicotomía mente-cerebro respecto a este complejo y sublime acto de la especie humana. Cuanto más complejo se vuelve el estudio de la consciencia, más frecuentes son los constructos hipotéticos, dado que las explicaciones van tomando distancia del mero funcionamiento neuronal y ganando consideración las propiedades emergentes.

No queda plenamente demostrado, como hemos comentado, que solamente las capacidades relacionadas con el funcionamiento del cerebro humano expliquen todos esos rasgos distintivos de la condición humana, como la capacidad de anticipar el futuro, de actuar en un mundo social complejo, como el conocimiento de uno mismo y el de los demás, el control sobre la propia existencia o la empatía.

Relacionado con la autoconciencia se halla el concepto de qualia, introducido por Daniel Dennett en 1990. Definido como el conjunto de propiedades fenomenológicas de la experiencia constituidas de elementos que sólo pueden ser conocidos desde el punto de vista del sujeto. Yo no puedo experimentar el dolor de otro, sólo puedo inferirlo, pero sin que exista transferencia directa de su experiencia. La tendencia de la actual neurociencia trata de explicar estos elementos cualitativos. En este sentido, el concepto de qualia se opone a lo mensurable, los quanta. Los qualia son estados subjetivos que, no teniendo ubicación alguna en un programa de ordenador, hacen imposible la comparación cerebro-ordenador. 
La neurociencia busca la unidad de la percepción, el constituyente mínimo de lo que llama conciencia, y la ha encontrado en los qualia, la sensación pura, la sensación de percibir un determinado color. Para Ramachandran es como si un extraterrestre que no tuviera receptores del color en los ojos pretendiera conocer "lo azul". Aun teniendo un concepto de lo azul, una teoría científica de cómo se captan y procesan en el cerebro los colores y midiendo nuestras respuestas nerviosas frente a los objetos que presentan la longitud de onda correspondiente, no podría hacerse idea de lo extraordinaria que resulta la experiencia real de ver el azul. Los qualia son irrevocables si hemos visto el color; podemos hablar de esta sensación en términos objetivos si se traduce el lenguaje de los impulsos nerviosos al lenguaje verbal; pero la experiencia del color, el sabor, el dolor, la auténtica percepción de estas cualidades es necesariamente subjetiva e incomunicable. Nadie que no la haya sentido entenderá por completo de qué hablamos. Los qualia inducen a pensar que la autoconciencia no es algo que puede ser estudiado por un observador externo sino que supone la interacción entre el observador y lo observado. Daniel Dennett identifica cuatro propiedades adscritas a los qualia:

-Inefables: no pueden ser comunicados o aprendidos por otros medios diferentes a la experiencia directa.

-Intrínsecos: propiedades no relacionales, que no cambian dependiendo de la relación de la experiencia con otras cosas.

-Privados: todas las comparaciones interpersonales de los qualia son sistemáticamente imposibles. 
-Directamente o inmediatamente aprehensibles en la conciencia: la experiencia de un quale es saber que uno experimenta un quale, y saber todo ello es saber acerca del quale.

\section{A modo de conclusión}

La conciencia se considera resultado de los de procesos neurobiológicos del cerebro, una propiedad derivada de su elevado nivel de complejidad. Sin embargo, la teoría de las propiedades emergentes, según la cual un sistema exhibe propiedades que no aparecen en los elementos aislados que lo compone, no restituye ad integrum el salto de lo biológico a la conciencia. En su centro de argumentación:

$$
x_{1}+x_{2}+\cdots+x_{n}+y=\mathrm{T}
$$

la $y$ representa ese algo más, sin origen determinado, que como propiedad mágica contribuye al todo T. Pero no está explicada y, por tanto, deja sin explicación la autoconciencia como propiedad emergente de un sistema como el cerebro humano, de tan alto grado de complejidad.

En nuestra exploración comprobamos, una vez más, que el debate pretende integrar la percepción en el método científico. Es un problema duro encajar el método científico — cuya condición objetiva sólo es aplicable a las regularidades de la naturaleza- a la consciencia, cuya naturaleza subjetiva representa una singularidad en la naturaleza. Desde que F. Crick y R. Penrose decidieran abordar desde el método científico el origen y desarrollo de la 
mente, una explosión de trabajos han presentado decenas de correlaciones entre áreas cerebrales y estados de consciencia, pero no se han verificado en el laboratorio ni la localización del yo - el que posee la experiencia- en el cerebro, ni los referidos modelos que plantean el procesamiento de información cerebral en la generación de la autoconsciencia. Encajar la consciencia en el método científico seguirá siendo un difícil problema académico, toda vez se acepten bases epistemológicas, como la del primer postulado bungiano, que niegan la existencia real de lo inmaterial. Los científicos saben que cada escala de observación muestra un mundo y presenta los efectos de dicha escala, y que hay efectos ausentes porque habitan otras escalas. El análisis de precisión microscópica no encuentra la frontera entre el cuerpo y la mente.

Apreciamos una deslealtad moderna en la pretensión de medir la experiencia interior con las formas de lo exterior, de tratar las sensaciones como símbolos o signos de la realidad y no como la realidad misma. La mente trabaja a base de representaciones y al mismo tiempo, el método científico obliga a tratar estas representaciones como signos.

Dice Javier Gomá que "la historia de la ciencia es un camino en ascensión que va levantando a su espalda una polvareda de ilustres falsedades" (Gomá, 2011). Es evidente que no podemos pensar sin generalizar, pero resulta obligado, en este cambio de época, una revisión profunda de las abstracciones. Nuestra civilización ha quedado atrapada en ellas, so riesgo de un destino fatal. Tal revisión es responsabilidad, no sólo de los filósofos de la naturaleza, también de la nueva ciencia de la cognición y, en todo caso, del diálogo fecundo entre ambas. En un tiempo en el que el esquema de abstracciones ha triunfado y facilitado el desarrollo tecnológico (que toma el relevo en la nue- 
va cultura), la ciencia no debe seguir guardando sepulcral silencio sobre aquello que más importa. A pesar de que podemos manipular farmacológicamente el cerebro para modificar la expresión de la conciencia, no logramos identificar su sustrato material. Explicar su naturaleza parece, en palabras de Juan Arana, "la cosa inexplicada por antonomasia, una causa incausada. Sin embargo, de ella depende la posibilidad de dar cuenta de la indagación más importante relativa al sentido de nuestra identidad”. Es evidente que la conciencia se ha enriquecido en el curso de la evolución, que su despliegue ha exigido a las estructuras orgánicas que la sustentan cambios continuos. Con la presente revisión no cabe más que concluir que el cerebro es fruto de la conciencia, y no al revés, por más que su correcto funcionamiento sea condición necesaria para que aquella se exprese en el mundo material. El problema difícil deja de serlo cuando se otorga a la consciencia un papel principal, en lugar de subordinarla al cerebro. Si uno posee libertad para decidir y si la plasticidad neuronal se reconfigura con cada experiencia y con cada aprendizaje (Davison 2003), entonces no es posible sostener que el cerebro fue primero.

\section{Bibliografia}

Arana, J., La Conciencia Inexplicada. Ensayo sobre los limites de la comprensión naturalista de la mente. Madrid, Biblioteca Nueva, 2015.

Allman, J. M. et al., "The von Economo neurons in frontoinsular and anterior cingulate cortex in great apes and humans". Brain StructFunct. Vol. 214, 495-517, 2010 
Armstrong, D. M., The mind-body problem: an opinionated introduction. Boulder, CO, Westview Press, 1999.

Bunge M., "Emergence and the mind". Neuroscience vol 2, Issue 4, 501-509, 1977

-Scientific realism: selected essays. Amherst, Prometheus Books, 2001.

-El problema mente-cerebro. Madrid, Tecnos, 2002.

-Chasing reality: strife over realism. Toronto, University of Toronto; 2006.

Caston, V., "Epiphenomenalisms, ancient and modern”. Philos Rev. Vol. 106, 309-63, 1997.

Chalmers, D. M., "El problema de la consciencia”. Investigacion y Ciencia. Vol. 2, 60-67, 1996.

Crick, F., La búsqueda científica del alma. Madrid. Debate, 1994.

Damasio, A. R., Descartes' error: emotion, reason, and the buman brain. New York, G.P. Putnam's Sons, 1994.

- La sensación de lo que ocurre: cuerpo y emoción en la construcción de la conciencia. Madrid, Debate, 2001.

Davison, R. J. et al., "Alterations in Brain and Immune Function Produced by Mindfulness Meditation”. Psychosomatic Medicine. Vol. 65, 564-570, 2003

Dennett, D., The intentional stance. Cambridge, MA, MIT Press, p. 43-68, 1987

-Quining Qualia. In Lycan W, ed. Mind and cognition. Oxford, Blackwell, p. 519-48, 1990

-Consciousness explained. Boston, Little, Brown \& Company, 1991.

Devitt, M., Realism and truth. Oxford, Blackwell, 1991.

Eccles, J. C.K and Popper, K. A., The self and its brain: an argument for interactionism. London, Routledge, 1984.

Edelman, G. M. and Tononi G., A universe of consciousness: how matter becomes imagination. New York, Basic Books, 2000.

Feigl H. Scriven, M. Maxwell, G. editors., Concepts, theories and the mind-body problem (Minnesota Studies in the Philosophy of Science, Volume 2). Minneapolis, University of Minnesota Press, 1967.

Fodor, J., The language of thought. New York, Crowell, 1975. 
Fulford, K. W. et al., The Oxford handbook of philosophyand psychiatry. Oxford, Oxford University Press, 2013.

Gazzaniga, M. The cognitive neurosciences. Cambridge, MA, MIT Press, 1995.

Gomá Lanzón J., Imitación y experiencia. Pre-Textos, 2003.

Gomá Lanzón J., Ingenuidad aprendida. Galaxia Gutenberg, 2011.

Lewis, D., “An argument for the identity theory”. J. Philos. Vol 63, 17-25, 1966.

Logothetis, N. K., "Single units and conscious vision". Philos Trans Royal Soc Lond B. 353, 1801, 1998.

McLaughlin, B. P., The Oxford handbook of philosophy of mind. Oxford, Clarendon Press, 2009

Llinás, R., El cerebro y el mito del yo. Ed. Norma, 2003.

Miller, A., Realism and antirealism. A handbook of philosophy of language. Oxford. Oxford University Press, p. 983-1005, 2006.

Putnam, H., Mind, language and reality. Cambridge, Cambridge University Press, 1975.

Place, U. T., Identifying the mind. New York, Oxford University Press, 2004.

Prigatano G., "Introduction". In Prigatano, G, eds. Awareness of deficit after brain injury. New York, Oxford University Press, p. 13, 1991.

Quian Quiroga, R. et al., "Invariant visual representation by single neurons in the human brain”. Nature 435, 1102-1107, 2005.

Ramachandran, V. S. Blakeslee S., Fantasmas en el cerebro. Madrid, Debate, 1999.

Reichenbach, H., The rise of scientific philosophy. Berkeley, University of California Press, 1973.

Russell, B., A history of Western philosophy. New York, Simon \& Schuster, 1961.

Smart, J. C., "Consciousness and awareness". Journal of Consciousness Studies 11, 41-50, 2004.

Stuss, D. T., "Disturbance of self-awareness after frontal system damage". In Prigatano G, eds. Awareness of deficit after brain injury. New York, Oxford University Press, p. 63, 1991.

Tarski, A., "The Semantic Conception of Truth". Philosophy and Phenomenological Research vol. 4, 1943. 


\section{La conciencia y el problema mente-cerebro}

Tulving, E., "Chronoesthesia: conscious awareness of subjective time". In Stuss DT, Knight R, eds. Principles of frontal lobe function. New York, Oxford University Press. pp 311325, 2002.

Van Fraassen, B. C., The scientific image. Oxford, Oxford University Press, 1980.

Wagensberg, J., Si la naturaleza era la pregunta, ¿̇cuál era la respuesta? Barcelona. Tusquet, 2012.

Esteban Fernández Hinojosa

Hospital Universitario Virgen del Rocío. Sevilla estebanfhinojosa@gmail.com 
